\title{
NOTE ON THE INTEGRATION OF A UNIFORMLY CONVERGENT SERIES THROUGH AN INFINITE INTERVAL.
}

BY PROFESSOR THOMAS S. FISKE.

[Read at the January meeting of the Society, 1897.]

IT is well known that if the terms of a uniformly convergent series

$$
u_{1}(x)+u_{2}(x)+\cdots+u_{n}(x)+\cdots
$$

are integrable through a finite interval $[a, b]$, the series defines a function $f(x)$ which is integrable through $[a, b]$, and its integral can be obtained by term by term integration, that is

$$
\int_{a}^{b} f(x) d x=\int_{a}^{b} u_{1}(x) d x+\cdots+\int_{a}^{b} u_{n}(x) d x+\cdots
$$

If, however, the interval considered is infinite, neither of these results is necessary.

Professor Osgood in his paper on uniform convergence and double limits, published in the Bulletin for November, illustrates by an example the case where such a series is non-integrable through an infinite interval. It may be of interest to some of the readers of his paper to see the following example of a uniformly convergent series which is integrable through an infinite interval, but the integral of which cannot be obtained by summing the integrals of its terms.

The series in question is*

$$
-2 x e^{-x^{2}}=\sum_{1}^{\infty}-2 x\left(\frac{1}{n^{2}} e^{-\frac{x^{2}}{n^{2}}}-\frac{1}{(n+1)^{2}} e^{-\frac{x^{2}}{(n+1)^{2}}}\right)
$$

We have

$$
\begin{gathered}
s_{n}(x)=-2 x e^{-x^{2}}+\frac{2 x}{(n+1)^{2}} e^{-\frac{x^{2}}{(n+1)^{2}}} \\
r_{n}(x)=-\frac{2 x}{(n+1)^{2}} e^{-\frac{x^{2}}{(n+1)^{2}}}
\end{gathered}
$$

* This series was obtained by making certain modifications in a series used by Darboux in his " Mémoire sur les fonctions discontinues," $A n$ nales de l'Ecole Normale, 2 series, vol. 4 (1875), p. 84. 


$$
\left|r_{n}(x)\right| \leqq \frac{\sqrt{2}}{(n+1) \sqrt{e}}
$$

The uniformity of convergence follows from the fact that the second member of the last relation is independent of $x$. By integrating this series term by term from 0 to $x$, we obtain

$$
\sum_{1}^{\infty}\left(e^{-\frac{x^{2}}{n^{2}}}-e^{-\frac{x^{2}}{(n+1)^{2}}}\right),
$$

which converges to

$$
e^{-x^{2}}-1=\int_{0}^{x}-2 x e^{-x^{2}} d x
$$

only when $x$ is finite, being equal to zero when $x$ is infinite.

In this connection it may be observed that the integration of an infinite series through an infinite interval is a question of triple limits. Uniformity of convergence on the part of the given series enables us to write

$$
\lim _{b=\infty} \int_{a}^{b} \lim _{n=\infty} s_{n}(x) d x=\lim _{b=\infty} \lim _{n=\infty} \int_{a}^{b} s_{n}(x) d x,
$$

but it does not enable us to write

$$
\lim _{b=\infty} \lim _{n=\infty} \int_{a}^{b} s_{n}(x) d x=\lim _{n=\infty} \lim _{b=\infty} \int_{a}^{b} s_{n}(x) d x .
$$

A sufficient condition for the latter relation would be the uniform convergence in the infinite interval of the series resulting from the term by term integration of the given series. This condition, however, has the theoretical disadvantage of not being immediately applicable to the given series. Jordan has given* a sufficient condition which is free from this disadvantage, viz., that $r_{n}(x)$ should be expressible in the form $\varepsilon_{n} \varphi(x), \varphi(x)$ being a positive function which has a finite integral in the infinite interval, and $\varepsilon_{n}$ converging uniformly to zero when $n$ approaches infinity.

Columbia University, January 30, 1897.

\footnotetext{
* Cours d'Analyse, 2 d edition, vol. 2 (1894), p. 62.
} 